\title{
Railroad Caller Districting with Reliability, Contiguity, Balance, and Compactness Considerations
}

\author{
Siyang Xie ${ }^{\mathrm{a}}$, Yanfeng Ouyang ${ }^{\mathrm{a}, *}$ \\ ${ }^{a}$ Department of Civil and Environmental Engineering, University of Illinois at Urbana-Champaign, Urbana, IL \\ 61801, USA
}

\begin{abstract}
Railroad companies rely on good call centers to reliably handle incoming crew/resource call demands so as to maintain efficient operations and customer services in their networks. This paper formulates a reliable caller districting problem which aims at partitioning an undirected network into a fixed number of districts. The demand of each district is assigned to crew caller desks (one primary desk, multiple backups) under possible desk disruption scenarios. We simultaneously take into account several operational criteria, such as district contiguity and compactness, workload balance, and caller desk service reliability. The resulted districting problem is modeled in the form of a complex mixed-integer program, and we develop a customized heuristic algorithm (based on constructive heuristic and neighborhood search) to provide near-optimum solutions in a reasonable amount of time. Hypothetical and empirical numerical examples are presented to demonstrate the performance and effectiveness of our methodology for different network sizes and parameter settings. Managerial insights are also drawn.
\end{abstract}

Keywords: districting, reliability, contiguity, workload balance, compactness

\section{Introduction}

Railroad companies regularly receive a large number of calls from their customers and business partners (normally via telephone, intercom, computer or other devices) regarding real-time train operation, service scheduling, and resource/crew arrangements in the field. The calls are typically intended for ensuring compliance with all railroad business rules and safety regulations of the Federal Railroad Administration (FRA). Some specific tasks of the calls include (i) contacting individual crew members and directing them to their assigned trains or job locations; and (ii) monitoring system operations, and collecting data and relevant information routinely across railroad divisions and subdivisions for internal and external reporting purposes. Such calls have significant implications on the operational efficiency of the railroad system and hence are important for the success of the railroad companies.

The call center serves as the information and decision hub for a railroad company's production and maintenance activities. The planning of the call center plays a critical role in the allocation and utilization of the railroad's manpower, equipment, and other resources. This is particularly the case when railroads are facing operational uncertainties and the risk of disruptions (e.g., due to adverse weather, train accidents, power outage, labor issues). In case of unexpected emergencies, effective communication and efficient resource (re-)allocation across the railroad network must be 
ensured via the call center such that backup plans for train timetabling, rolling stock and crew scheduling can be carried out in time - The call center itself must be functioning reliably so as to be responsible for real-time emergency management and disaster response. Therefore, a good call center design that can reliably and efficiently handle incoming calls across the railroad system is very critical.

Usually a call center for a railroad company consists of multiple crew caller desks, each of which is responsible for the calls from a particular predefined spatial region (i.e., district). A good call center design should have the following characteristics: (i) all incoming call demand can be handled by a properly assigned caller desk under any circumstance, otherwise significant penalty will occur due to disruption to railroad operations; (ii) the expected workload is well balanced across caller desks, so that no desks are too much more occupied than others; (iii) the spatial district served by a caller desk should be contiguous so as to satisfy a number of practical operational requirements, e.g., administrative autonomy for resource/crew reallocation and train traffic management; and (iv) the spatial district corresponding to one caller desk is compact in shape so as to avoid high transportation/logistics costs inside odd-shaped districts.

With all these practical considerations, the railroad call center design problem is closely related to a geographical districting problem. Districting is a well-known problem in the operations research literature. It aims at partitioning a geographical space into sub-districts under various criteria and constraints. Depending on the specific application context, operational criteria may include the district contiguity, district compactness, workload balance, socio-economic homogeneity, etc. In the literature, probably the most intensively studied problem is regarding political districting, which divides a jurisdiction area (e.g., a state or a region) into electoral constituencies such that the political candidates from each area are elected to a parliamentary assembly. The "one man-one vote" principle requires that all districts contain approximately the same number of candidates/voters to avoid benefiting a certain party or candidate. Hess et al. (1965) is among the first several that used mathematical programming techniques to model the political districting problem. An assignment formulation with additional planning constraints was developed and an iterative heuristic algorithm was proposed. But the convergence of the algorithm or the contiguity of districts is not guaranteed. Garfinkel and Nemhauser (1970), on the other hand, considered selecting districts from a set of predefined feasible ones. The various constraints (e.g., contiguity, compactness) were implemented while defining the set of feasible regions (before implementing the optimization model). Mehrotra et al. (1998) adopted a column generation method to solve a similar problem as the one in Garfinkel and Nemhauser (1970). Bozkaya et al. (2003) considered more operational criteria, which were evaluated and incorporated as soft constraints. Health services districting is another application context that aims at partitioning a health service territory into districts and assign a certain amount of medical resources to each district. Blais et al. (2003) studied the districting for a public health clinic where five districting criteria were sought under a tabu search algorithm. School districting problem assigns residential neighborhoods to existing schools, as different important criteria for planning must be taken into account, e.g., the capacity, the accessibility, and the racial balance of each school. Notably, Ferland and Guénette (1990) proposed a decision support system to solve the school districting problem; the system included a network-based mathematical model and used several heuristic procedures to assign network edges (with students located on it) to schools. Other examples of districting problems include sales/market districting (Hess and Samuels, 1971), police districting (Camacho-Collados et al., 2015), waste/garbage collection districting (Muyldermans et al., 2002), etc.

All these traditional districting problems simply focus on partitioning a network/area into districts under some operational considerations. However, most of these studies ignore the fact that in many application contexts, the partitioned districts must be assigned to a supplier/facility, e.g., 

always functioning and thus no backup assignments are needed. Recently, the reliability of service providers (caused by internal or external factors) has caught considerable attention. When a facility fails, all the demands originally assigned to this facility have to either be reassigned to another surviving facility or lose service. In the recent years, many studies started to model probabilistic disruptions, Snyder and Daskin (2005) considered that facilities are subject to independent probabilistic disruptions with identical failure probabilities. Berman et al. (2007) formulated a nonlinear mixed-integer programming model for the reliable location model and designed an efficient heuristic algorithm. Chen et al. (2011) studied a reliable joint inventory-location problem where facilities are subject to disruption risks. In the railroad caller districting problem, the caller desks, which play the role of facilities, are also subject to failure due to technical and personnel reasons. To the best of our knowledge, no work has been done to incorporate service reliability issues into the districting problem. Moreover, it is worth noting that under reliability issues, the traditional modeling methods for addressing various operational criteria (e.g., workload balancing, district compactness, and contiguity) would no longer work - new customized treatment methods are needed.

In light of these challenges, in this paper, we formulate the reliable caller districting problem as a mixed-integer programming model. A series of modeling techniques (e.g., network flow constraints) are adopted to address some of the practical planning criteria: (i) each district must be contiguous; (ii) each district is compact in shape; (iii) workload is balanced across districts. The reliability of caller desk service is also incorporated by introducing reliable desk re-assignments. Note that when desks are subject to disruptions, the expected workload of each district across all possible desk failure scenarios should be considered. Customized solution approach consisting of constructive and neighborhood search heuristics are developed to efficiently solve the mathematical model. Several numerical examples including a series of hypothetical test cases and an empirical full-scale railroad case study are conducted to demonstrate the performance and applicability of our methodology. Various managerial insights are also drawn.

The remainder of the paper is organized as follows. Section 2 introduces the various operational criteria and formulates the reliable caller districting problem into a mixed-integer mathematical model. Section 3 presents the customized solution approaches to efficiently solve the problem. In Section 4, results for both the hypothetical and empirical examples are presented. Finally, Section 5 concludes the paper and discusses future research directions.

\section{Methodology}

In this section, we present a mixed-integer linear programming model for the railroad caller districting problem. The challenge is essentially to partition a given undirected network $G=(I, E)$ (with node set $I$ and edge set $E$ ) into a fixed number of subgraphs, which we call districts, that jointly cover all the nodes in $I$, and to assign these districts to caller desks for service. Binary parameter $\delta_{i_{1} i_{2}}$ indicates whether two nodes $i_{1} \in I$ and $i_{2} \in I$ are adjacent in network $G$; i.e., $\delta_{i_{1} i_{2}}=1$ if link $\left(i_{1}, i_{2}\right) \in E$, or $\delta_{i_{1} i_{2}}=0$ otherwise. Each node in the graph, $i \in I$, generates a certain quantity of crew service demand $D_{i}$ (e.g., crew dispatch and emergency calls). Let $J=\{1,2, \cdots,|J|\}$ be the set of district indices. The nodes in each district, $I_{j}, j \in J$, must be associated with one caller desk so that the desk is primarily in charge of serving the demand from that district.

Meanwhile, we also consider several operational criteria and constraints, including district contiguity and compactness, caller desk service reliability, and workload balance. 


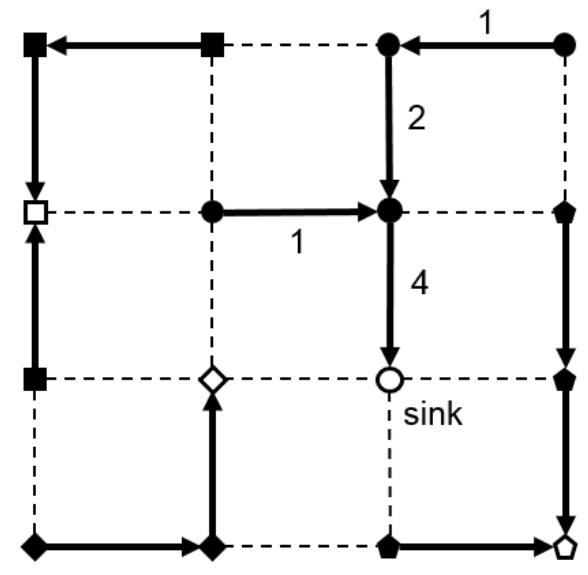

(a) Feasible

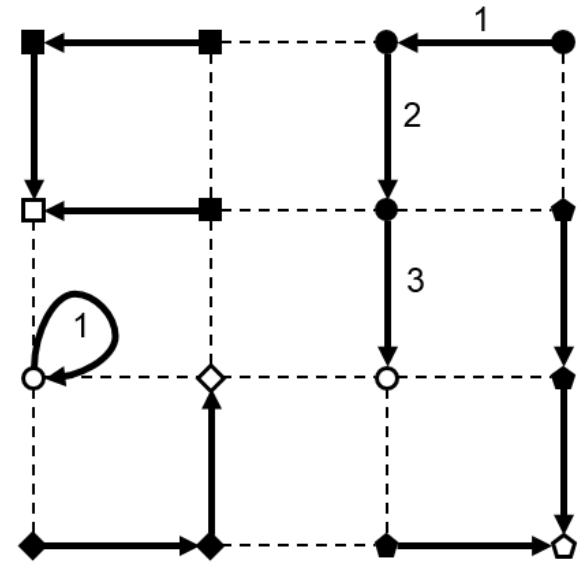

(b) Infeasible

Figure 1: Network flow for contiguity.

The idea is actually quite simple. For each of the $|J|$ caller desks, we imagine that the network $G$ contains a corresponding "proxy" sink node that each can absorb any amount of incoming flow. We also assume that one unit of virtual flow is generated at each node $i \in I$, and it will flow through the network to finally reach one of the $|J|$ sink nodes. If each district contains exactly one sink node to receive all the virtual flow that has originated within the district, and if we can force that no flow is allowed between different districts, then we have successfully partitioned $G$ into several mutually disjoint but connected districts. Figure 1(a) illustrates a feasible network partition with four districts, and an associated virtual flow pattern. In the figure, each arrow indicates the virtual flow between nodes, and the number near the arrow gives the flow volume. The nodes in each partitioned district have the same shape (e.g., circle, square), while the sink node of this district is hollow. As shown, a partition is feasible if all virtual flow from a district could be collected and routed to its sink without passing any nodes outside of the district. Figure 1(b), in contrast, illustrates an infeasible partition, because there is no way to collect and route virtual flow from all "circle" nodes to a single sink node in this district, i.e., the district is not contiguous.

For any $i \in I, j \in J$, we let variable $y_{i j}=1$ if node $i$ is in district $j$, or $y_{i j}=0$ otherwise. In addition, we let $x_{i j}=1$ if node $i$ is the sink node of district $j$, or $x_{i j}=0$ otherwise. We further let $z_{i_{1} i_{2}}^{j}=1$ if nodes $i_{1}$ and $i_{2}$ are adjacent in network $G$ and both of them are in district $j$, or $z_{i_{1} i_{2}}^{j}=0$ otherwise. The virtual link flow from any node $i_{1}$ to any node $i_{2}$ is denoted as $f_{i_{1} i_{2}}^{j}, \forall i_{1}, i_{2} \in I$. The contiguity of district $j \in J$ is then ensured by the following constraints:

$$
\begin{aligned}
& \sum_{j \in J} y_{i j}=1, \forall i \in I, \\
& \sum_{i \in I} x_{i j}=1, \forall j \in J,
\end{aligned}
$$




$$
\begin{aligned}
& x_{i j} \leq y_{i j}, \forall i \in I, j \in J, \\
& z_{i_{1} i_{2}}^{j} \leq \delta_{i_{1} i_{2}} \cdot \frac{y_{i_{1} j}+y_{i_{2} j}}{2}, \forall i_{1}, i_{2} \in I, j \in J,
\end{aligned}
$$$$
f_{i_{1} i_{2}}^{j} \leq(|I|-|J|) z_{i_{1} i_{2}}^{j}, \forall i_{1}, i_{2} \in I, j \in J
$$$$
y_{i_{1} j}+\sum_{i_{2} \in I} f_{i_{2} i_{1}}^{j} \geq \sum_{i_{2} \in I} f_{i_{1} i_{2}}^{j}, \forall i_{1} \in I, j \in J
$$$$
y_{i_{1} j}+\sum_{i_{2} \in I} f_{i_{2} i_{1}}^{j} \leq \sum_{i_{2} \in I} f_{i_{1} i_{2}}^{j}+(|I|-|J|) x_{i_{1} j}, \forall i_{1} \in I, j \in J .
$$

Constraints (1a) enforce that each node $i \in I$ belongs to exactly one district. Constraints (1b) make sure that each district has exactly one sink node. Constraints (1c) ensure that the sink node of each district should be inside the district due to the contiguity requirement. Constraints (1d) and (1e), together with integrality of the respective decision variables, require that virtual flow only exists on links within each district, while $|I|-|J|$ is the maximum possible flow volume on a link. Constraints (1f) and (1g) stipulate flow conservation at nodes in the network, except for those sink nodes (i.e., any node $i \in I$ with $x_{i j}=1$ ).

\subsection{Reliable Desk Assignment}

Due to exogenous reasons (such as adverse weather, power outage, etc.), each crew caller desk is subject to independent disruptions with an identical probability $q$, which is defined as the fraction of time in a relatively long horizon (e.g., a year) during which a caller desk is in a disrupted state (e.g., due to adverse weather or labor issues). Once a caller desk fails, all its demands (i.e., service calls) are either served by another functioning backup caller desk, or the service is lost. For each district $j \in J$, we plan a series of backup plans that involve up to $R \geq 1$ other caller desks. We call its $r$ th choice of caller desk as its level- $r$ choice, while the level-0 choice is its original associated caller desk $j$. The district receives service from its level- $r$ caller desk if its level-0, $\cdots$, level- $(r-1)$ choices have all become unavailable, which occurs with a corresponding probability of $(1-q) q^{r}$. To the very extreme, if all of the district's $R+1$ assigned caller desks (including the level-0 choice) have failed, which occurs with a probability of $q^{R+1}$, every unit of demand in the district will incur a penalty of $W_{\text {missing. }}$. Without loss of generality, we construct a dummy caller desk and assign all "lost" demand to this dummy desk.

We let variable $Y_{j k}^{r}=1$ if district $j \in J$ is assigned to caller desk $k$ at level $r$, or $Y_{j k}^{r}=0$ otherwise. Similarly, let $Y_{j 0}^{r}=1$ if district $j \in J$ is assigned to the dummy caller desk at level $r$, or $Y_{j 0}^{r}=0$ otherwise. The following constraints enforce such back-up assignments to caller desks:

$$
\begin{aligned}
& \sum_{k \in J, k \neq j} Y_{j k}^{r}+Y_{j 0}^{r}=1, \forall j \in J, r=1,2, \cdots, R, \\
& Y_{j 0}^{r} \leq Y_{j 0}^{r+1}, \forall j \in J, r=1,2, \cdots, R, \\
& Y_{j 0}^{R+1}=1, \forall j \in J .
\end{aligned}
$$

Here, constraints (2a) ensure that at each backup level, each district is assigned to either a regular caller desk or the dummy desk. Constraints $(2 \mathrm{~b})$ enforce that if a district is assigned to the dummy desk at some level $r$, it is assigned to the dummy desk at all higher levels $r+1, \cdots, R+1$. Constraints (2c) guarantee that all districts are assigned to the dummy desk at level $R+1$.

Even in a desk failure scenario, the continuity requirement mandates that any districts (re)assigned to a functioning desk should still be a connected graph. To ensure this, we enforce a strong requirement that if district $j$ can be re-assigned to a backup desk $k$, then districts of indices 
$j$ and $k$ must be adjacent to each other, i.e., there exist two nodes $i_{1} \in I_{j}$ and $i_{2} \in I_{k}$ such that $\delta_{i_{1} i_{2}}=1$. With this restriction, if a district has a limited number of neighbors, we may not be able to assign a district $j \in J$ to a regular caller desk at some level $r \leq R$; instead, the district will be re-assigned to the dummy desk with probability $q^{r}$. As such, the probability that $j$ is assigned to the dummy desk is calculated as $\sum_{s=r}^{R}(1-q) q^{s}+q^{R+1}=q^{r}$, which equals to the true probability for service loss. We define $w_{i_{1} i_{2}}^{j_{1} j_{2}}=1$ if nodes $i_{1}$ and $i_{2}$ belong respectively to districts $j_{1}$ and $j_{2}$, or $w_{i_{1} i_{2}}^{j_{1} j_{2}}=0$ otherwise. Furthermore, we define $l_{j k}=1$ if districts $j$ and $k$ are connected, and 0 otherwise. Then, the contiguity requirement can still be enforced under caller desk disruption and district reassignment scenarios by the following additional constraints and the respective integralities of decision variables:

$$
\begin{aligned}
& w_{i_{1} i_{2}}^{j_{1} j_{2}} \leq \delta_{i_{1} i_{2}} \cdot \frac{y_{i_{1} j_{1}}+y_{i_{2} j_{2}}}{2}, \forall i_{1}, i_{2} \in I, j, k \in J, \\
& l_{j k} \leq \sum_{i_{1} \in I} \sum_{i_{2} \in I} w_{i_{1} i_{2}}^{j k}, \forall j, k \in J, \\
& \sum_{r=1}^{R} Y_{j k}^{r} \leq l_{j k}, \forall j, k \in J .
\end{aligned}
$$

Constraints (3a) and (3b) determine whether two districts $j \in J$ and $k \in J$ are adjacent to each other. Constraints (3c) enforce that a district can only be re-assigned to an adjacent regular caller desk.

\subsection{Workload Balance}

We aim at balancing the expected total demands assigned to each caller desk across all normal and disruption scenarios. It is easy to see that such expected demand for a regular desk $k \in J, X_{k}$, can be derived as follows:

$$
X_{k}=\sum_{i \in I} y_{i k} D_{i}(1-q)+\sum_{j \in J} \sum_{r=1}^{R} Y_{j k}^{r} \sum_{i \in I} y_{i j} D_{i}(1-q) q^{r}, \forall k \in J .
$$

The first and second terms are respectively the expected cost of serving the demand in district $k$ via desk $k$ and all possible re-assignments. Similarly, the expected demand assigned to the dummy desk can be expressed as follows:

$$
X_{0}=\sum_{j \in J} Y_{j 0}^{R+1} \sum_{i \in I} y_{i j} D_{i} q^{R+1}+\sum_{j \in J} \sum_{r=1}^{R} Y_{j 0}^{r} \sum_{i \in I} y_{i j} D_{i}(1-q) q^{r}
$$

A simple way to balance the workload is to minimize the maximum value of $\left\{X_{j}\right\}_{j \in J}$, i.e., $X_{\max }:=\max _{j \in J} X_{j}$. Note that the following constraints hold:

$$
X_{\max } \geq X_{j}, \forall j \in J
$$

\subsection{Compactness}

The compactness of districts prevents high crew operating costs within oddly-shaped districts. High compactness indicates that any district should be circular or square in shape rather than being elongated. In our reliable caller districting problem, due to possible failures of desks, a demand may be serviced by different desks at different distances. Therefore, we define the "expected" compactness of a district $j$ based on two levels of spatial hierarchy: (i) the "median"-type total 
weighted distance for all nodal demand within district $j$ to its choice-0 desk (i.e., the sink within the district), and (ii) the expected distance from the "own" sink node of district $j$ to all other "backup" sink nodes at different choice levels. For a district $j \in J$, we define $v_{i_{1} i_{2}}^{j}=1$ if $i_{1} \in I_{j}$ and $i_{2}$ is the sink node of district $j$, or $v_{i_{1} i_{2}}^{j}=0$ otherwise. We also denote the network shortest path distance between nodes $i_{1}$ and $i_{2}$ as $d_{i_{1} i_{2}}$. The shortest path distance between the sink nodes of two districts $j$ and $k$, which is a decision variable that depends on the corresponding sink locations, is denoted as $\hat{d}_{j k}$. We know the following must hold:

$$
\begin{aligned}
& v_{i_{1} i_{2}}^{j} \geq y_{i_{1} j}+x_{i_{2} j}-1, \forall i_{1}, i_{2} \in I, \\
& \hat{d}_{j k} \geq d_{i_{1} i_{2}}\left(x_{i_{1} j}+x_{i_{2} k}-1\right), \forall i_{1}, i_{2} \in I, j, k \in J .
\end{aligned}
$$

Constraints (7a) determine whether a node $i_{1} \in I$ and a sink $i_{2} \in I$ are in the same district. Constraints $(7 \mathrm{~b})$ compute the distance between the sink nodes of two districts $j$ and $k$. Then the compactness measure for district $j$ is calculated as

$$
C_{j}=\sum_{i_{1} \in I} \sum_{i_{2} \in I} d_{i_{1} i_{2}} v_{i_{1} i_{2}}^{j}+\alpha \sum_{k \in J} \sum_{r=1}^{R}(1-q) q^{r} Y_{j k}^{r} \hat{d}_{j k}, \forall j \in J,
$$

where $\alpha$ is a weight parameter. A larger value of $C_{j}$ indicates a less compact district $j$.

\subsection{Formulation}

Now, the reliable caller districting problem (RCD) can be formulated as the following mixedinteger programming model:

$$
\begin{aligned}
(\mathrm{RCD}) \min & W_{\text {balance }} \cdot X_{\max }+W_{\text {compact }} \cdot \sum_{j \in J} C_{j}+W_{\text {missing }} \cdot X_{0} \\
\text { s.t. } \quad & (1 \mathrm{a})-(1 \mathrm{~g}),(2 \mathrm{a})-(2 \mathrm{c}),(3 \mathrm{a})-(3 \mathrm{c}),(4),(5),(6),(7 \mathrm{a})-(7 \mathrm{~b}),(8), \\
& y_{i j}, x_{i j}, z_{i_{1} i_{2}}^{j}, w_{i_{1} i_{2}}^{j_{1} j_{2}}, l_{j k}, v_{i_{1} i_{2}}^{j}, Y_{j k}^{r}, Y_{j 0}^{r} \in\{0,1\}, f_{i_{1} i_{2}}^{j}, \hat{d}_{j k} \geq 0, \\
& \forall i, i_{1}, i_{2} \in I, j, k \in J, r=1,2, \cdots, R,
\end{aligned}
$$

where $W_{\text {balance }}, W_{\text {compact }}$ and $W_{\text {missing }}$ are the relative weight coefficients for the maximum desk workload $X_{\max }$, the compactness measure $C_{j}$, and the dummy desk workload $X_{0}$, respectively. The objective function (9a) presents the expected system cost including the "cost" for workload balancing, the "cost" for compactness, and the penalty for demand loss. We assume that $W_{\text {missing }}>$ $W_{\text {balance}}$, otherwise the problem becomes trivial such that all districts will be assigned to the dummy desk at level 1 and no further backup plan is needed. In addition to Constraints (1a) - (8), Constraints (9b) enforce the integrity and non-negativity of all decision variables.

The formulation (RCD) contains several nonlinear terms $Y_{j k}^{r} y_{i j}, Y_{j 0}^{r} y_{i j}$ and $Y_{j k}^{r} \hat{d}_{j k}$ in several sets of constraints. We linearize them by applying a variant of the technique introduced by Sherali and Alameddine (1992), whereas $Y_{j k}^{r} y_{i j}, Y_{j 0}^{r} y_{i j}$ and $Y_{j k}^{r} \hat{d}_{j k}$ are replaced by new continuous variables 
$U_{j k}^{i r}, U_{j 0}^{i r}$ and $U_{j k}^{r}$, respectively. Their equivalence is enforced by adding the following new constraints.

$$
\begin{aligned}
& U_{j k}^{i r} \geq Y_{j k}^{r}+y_{i j}-1, \forall j, k \in J, i \in I, r=1,2, \cdots, R, \\
& U_{j k}^{i r} \leq Y_{j k}^{r}, \forall j, k \in J, i \in I, r=1,2, \cdots, R, \\
& U_{j k}^{i r} \leq y_{i j}, \forall j, k \in J, i \in I, r=1,2, \cdots, R, \\
& U_{j 0}^{i r} \geq Y_{j 0}^{r}+y_{i j}-1, \forall j \in J, i \in I, r=1,2, \cdots, R+1, \\
& U_{j 0}^{i r} \leq Y_{j 0}^{r}, \forall j \in J, i \in I, r=1,2, \cdots, R+1, \\
& U_{j 0}^{i r} \leq y_{i j}, \forall j \in J, i \in I, r=1,2, \cdots, R+1, \\
& U_{j k}^{r} \geq \hat{d}_{j k}+d_{\max }\left(Y_{j k}^{r}-1\right), \forall j, k \in J, r=1,2, \cdots, R, \\
& U_{j k}^{r} \leq \hat{d}_{j k}, \forall j, k \in J, r=1,2, \cdots, R, \\
& U_{j k}^{r} \leq d_{\max } Y_{j k}^{r}, \forall j, k \in J, r=1,2, \cdots, R, \\
& U_{j k}^{i r_{1}}, U_{j 0}^{i r_{2}}, U_{j k}^{r_{1}} \geq 0, \forall j, k \in J, i \in I, r_{1}=1,2, \cdots, R, r_{2}=1,2, \cdots, R+1,
\end{aligned}
$$

where $d_{\max }$ is the maximum distance between any two nodes in network $G$. Note from (10a)-(10c) that $U_{j k}^{i r}=1$ if and only if $Y_{j k}^{r}=y_{i j}=1$; if either $Y_{j k}^{r}$ or $y_{i j}$ equals 0 , then $U_{j k}^{i r}=0$ as well. Hence, these constraints ensure that $U_{j k}^{i r}=Y_{j k}^{r} y_{i j}$ exactly. Similarly, (10d)-(10f) ensure $U_{j 0}^{i r}=Y_{j 0}^{r} y_{i j}$. From (10g)-(10i), and the fact that $\hat{d}_{j k}-d_{\max } \leq 0 \leq \hat{d}_{j k} \leq d_{\max }$, we know that $U_{j k}^{r}=\hat{d}_{j k}$ if $Y_{j k}^{r}=1$, or 0 otherwise. These constraints exactly function in the same way as $U_{j k}^{r}=\hat{d}_{j k} Y_{j k}^{r}$. The model formulation (RCD) is then transformed into the following linearized reliable caller districting problem (LRCD):

$$
\begin{array}{ll}
(\mathrm{LRCD}) \min \quad & W_{\text {balance }} \cdot X_{\max }+W_{\text {compact }} \cdot \sum_{j \in J} C_{j}+W_{\text {missing }} \cdot X_{0} \\
\text { s.t. } \quad & (1 \mathrm{a})-(1 \mathrm{~g}),(2 \mathrm{a})-(2 \mathrm{c}),(3 \mathrm{a})-(3 \mathrm{c}),(6),(7 \mathrm{a})-(7 \mathrm{~b}),(9 \mathrm{~b}), \\
& (10 \mathrm{a})-(10 \mathrm{j}), \\
& X_{k}=\sum_{j \in J} \sum_{r=1}^{R} \sum_{i \in I} U_{j k}^{i r} D_{i}(1-q) q^{r}+\sum_{i \in I} y_{i k} D_{i}(1-q), \forall k \in J, \\
& X_{0}=\sum_{j \in J} \sum_{r=1}^{R} \sum_{i \in I} U_{j 0}^{i r} D_{i}(1-q) q^{r}+\sum_{j \in J} \sum_{i \in I} U_{j 0}^{i R+1} D_{i} q^{R+1}, \\
& C_{j}=\sum_{i_{1} \in I} \sum_{i_{2} \in I} D_{i_{1} i_{2}} v_{i_{1} i_{2}}^{j}+\sum_{k \in J} \sum_{r=1}^{R} U_{j k}^{r}(1-q) q^{r}, \forall j \in J .
\end{array}
$$

\section{Solution Approach}

The mixed-integer linear program (LRCD) could be potentially solved by commercial solvers such as CPLEX or Gurobi. However, as we will show in the case studies in Section 4, the computational burden is greatly exacerbated by the network-flow based constraints and the reliable assignment strategy, especially when the network size is relatively large. It takes a large amount of computation time for the solvers to obtain even a feasible solution (usually with a quite poor quality). In light of this challenge, in this section, we develop a customized heuristic algorithm to obtain near-optimum solutions in a reasonable amount of time. The algorithm contains two parts: 
(i) a constructive heuristic to obtain an initial solution, and (ii) a neighborhood search heuristic to improve the solution.

\subsection{Constructive heuristic}

We propose a constructive heuristic to obtain an initial solution. We first define the deterministic version (caller desks are always functioning) of the linearized caller districting problem as follows:

$$
\begin{aligned}
(\mathrm{DLRCD}) \min \quad & W_{\text {balance }} \cdot X_{\max }+W_{\text {compact }} \cdot \sum_{j \in J} C_{j} \\
\text { s.t. } \quad & (1 \mathrm{a})-(1 \mathrm{~g}), \\
& X_{j}=\sum_{i \in I} y_{i j} D_{i}, \forall j \in J, \\
& X_{\max } \geq X_{j}, \forall j \in J, \\
& v_{i_{1} i_{2}}^{j} \geq y_{i_{1} j}+x_{i_{2} j}-1, \forall i_{1}, i_{2} \in I, j \in J, \\
& C_{j}=\sum_{i_{1} \in I} \sum_{i_{2} \in I} d_{i_{1} i_{2}} v_{i_{1} i_{2}}^{j}, \forall j \in J .
\end{aligned}
$$

In this problem, the operational requirements including district contiguity, workload balance, and district compactness are all considered, so it is likely to generate an initial solution with a reasonably good quality. The detailed steps of the constructive heuristic to solve DLRCD are described as follows:

Step 1. Solve a median problem where $|J|$ sinks are initially located at selected nodes in the network $G$. Note that the districts in the solution of the median problem are not necessarily contiguous due to workload balancing.

Step 2. Starting with the $|J|$ initial sink nodes, we expand them into a set $J$ of contiguous districts as follows:

(i) Compute the average demand of each district as $\bar{D}=\sum_{i \in I} D_{i} /|J|$, and set a demand threshold $\widehat{D}$ as a function of $\bar{D}$, e.g., $\widehat{D}=\beta \bar{D}$, or $\widehat{D}=\bar{D}+$ Constant;

(ii) For each district $j \in J$ with demand $\sum_{i \in I_{j}} D_{i}$, if there exists one node $i$ such that $i$ is adjacent to $j$ and $\sum_{i^{\prime} \in I_{j}} D_{i^{\prime}}+D_{i} \leq \widehat{D}$, we expand district $j$ by attaching node $i$ and the associated links to it;

(iii) If no district can be further expanded without violating the workload limit, but there remain some nodes that have not been included in any district, do the following. For each remaining node $i$, we try to attach it to each of its adjacent districts and evaluate the resulting workload balance; select the attachment that yields the least workload balance violation.

Step 3. Given the set $J$ of districts generated in Step 2, we check for any pair of districts $j$ and $k$, if they are adjacent and the difference between their demands exceeds a specified threshold $\widetilde{D}$ (e.g., $\widetilde{D}=\gamma \bar{D}$ ), we run DLRCD to re-partition the network with districts other than $j$ and $k$ fixed.

This process continues until there exists no such pair of districts, or when some other termination criteria are met, e.g., the maximum number of iterations is reached. 


\subsection{Neighborhood search}

The solution obtained by the constructive heuristic does not consider the possible failures of caller desks. To incorporate the reliability issue into the solution, we develop a neighborhood search heuristic. In the solution space, a neighborhood of a district partition solution is defined as another partition with two of the adjacent districts redesigned (i.e., the nodes in the two districts are reassigned to yield two new districts) while all other districts remain the same.

Specifically, given the set $J$ of districts, if districts $j$ and $k$ are to be redesigned, we let $L=$ $J \backslash\{j, k\}$, fix the following variables, and solve LRCD again to re-partition the network:

(i) $x_{i j}, y_{i j}, z_{i_{1} i_{2}}^{j}, f_{i_{1} i_{2}}^{j}, v_{i_{1} i_{2}}^{j}, \forall i, i_{1}, i_{2} \in I, j \in L$;

(ii) $w_{i_{1} i_{2}}^{j_{1} j_{2}}, \forall j_{1}, j_{2} \in L$;

(iii) $l_{j k}, \hat{d}_{j k}, \forall j, k \in L$;

After solving the LRCD model, if the new solution is feasible and yields an improvement to the objective, it will be accepted and the partition solution will be updated. Then the set of possible neighborhood moves is updated and the neighborhood search process starts again. The searching procedure continues until some stopping criteria are met, or if all possible neighborhood moves have been enumerated without yielding any improvements.

The above algorithm re-designs two neighboring districts at a time. In general, any number of districts can be redesigned together in one search move. Since the assignment of demand in each district is interrelated with decisions in many other districts, re-partitioning multiple districts in one move may lead to a better solution or help avoid a local optimum. However, in so doing, the corresponding LRCD in each move takes a longer time to solve. So we set a max time for each move, taking into account the tradeoff between the improvement of solution quality and the computation time in each move.

\section{Case Study}

We apply the mathematical model and solution approaches to two examples so as to demonstrate their applicability and performance. In the first example, we partition a series of hypothetical grid networks of various sizes (as shown in Figure 2(a)), and compare the performance of different solution approaches. The second numerical example, on the other hand, involves a full-scale caller districting problem for a U.S. Class I railroad company (as shown in Figure 2(b)). The proposed model and solution algorithms are programmed in C\# and run on a 64-bit Intel i7-3770 computer with 3.40 GHz CPU and 8G RAM. The mixed-integer linear programs, if solved directly, are tackled by commercial solver Gurobi using up to 4 threads.

\subsection{Hypothetical grid networks}

For each value $n \in\{4,5,6,7,8\}$, an $n \times n$ grid network is generated to represent a hypothetical study region with $n^{2}$ nodes and $2 n(n-1)$ links. The $n^{2}$ nodes are indexed as $1,2, \cdots, n^{2}$ from left to right, and from bottom to top, as shown in Figure 2(a). The length of every edge between two adjacent nodes is set to 1 . For node $i$, the demand is set to $30+20(\bmod (i, 10) \cdot 10 \pi / 13)$. The failure probability of each caller desk is assumed to be 0.1 . We allow the number of districts $|J| \in\{5,7\}$, and the maximum assignment level $R \in\{3,4\}$. The penalty values are set as $W_{\text {balance }}=$ $1.0, W_{\text {compact }}=2.0$, and $W_{\text {missing }}=500.0$, respectively.

We use two different solution approaches to solve the reliable districting problems for these grid networks. The first approach directly applies Gurobi to solve the linearized programming models 


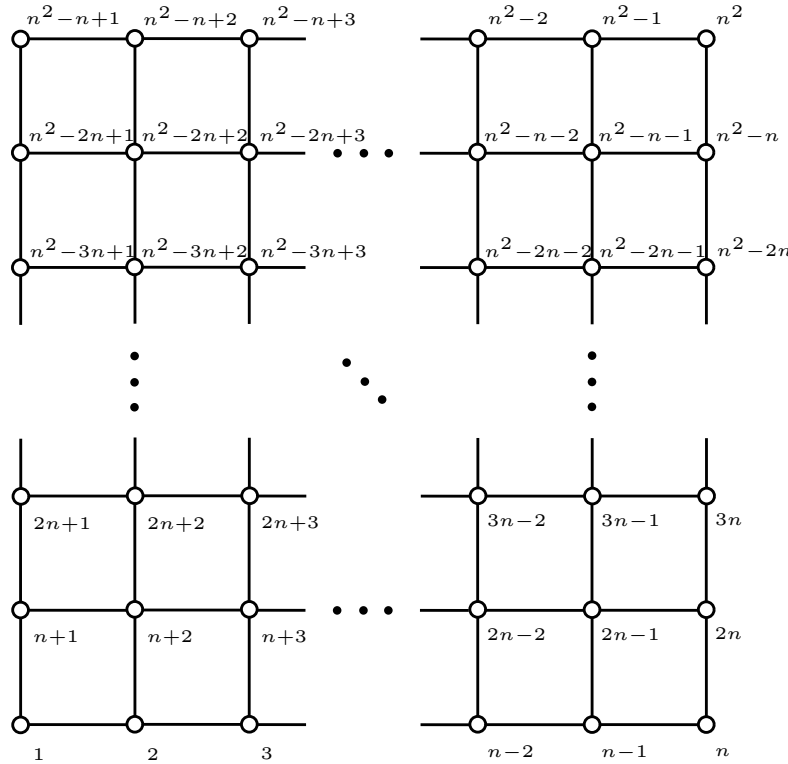

(a) $n \times n$ grid network

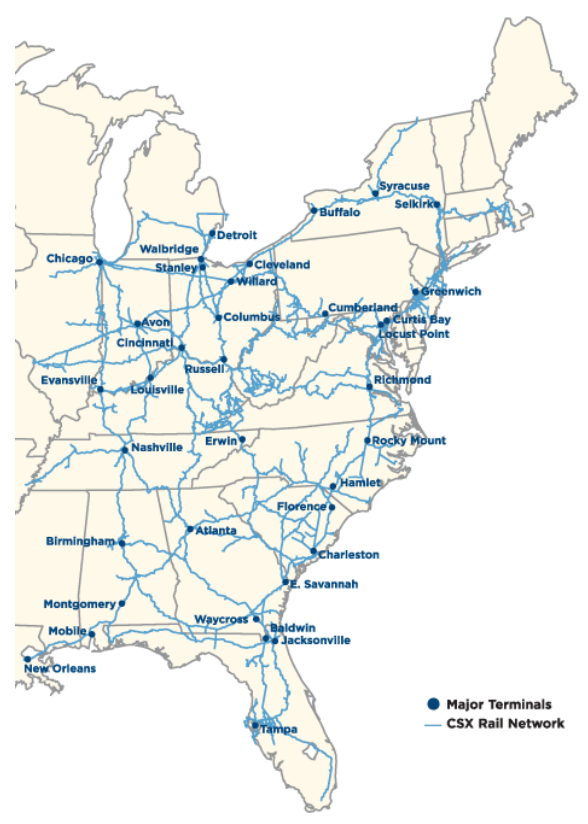

(b) The railroad network

Figure 2: Spatial networks used in the case study section.

(11a) - (11d), while the computation time limit is set to 7200s. The second approach uses the proposed heuristic algorithm (i.e., construction and neighborhood search), the max time limit for each search move is set to 60s. Table 1 summarizes and compares the results obtained by the two approaches.

Overall, as the network size, the number of partitions, and/or the maximum number of backup levels $R$ increase, the problem becomes more challenging to solve. Gurobi seems to have difficulty closing the reported optimality gap for all the test cases within the 7200s computation time limit, while for all $8 \times 8$ cases, Gurobi even could not find a feasible solution. In contrast, our proposed heuristic approach is able to obtain reasonable solutions in a relatively short amount of computation time. By comparing the results obtained by the two approaches, we observe that their solutions are comparable only for moderate size problems (e.g., $n=4,5$ partitions, and $R=3$ or 4 ). When the problem size is large, the quality of the Gurobi solution is quite poor, i.e., its reported gap is very large and its best feasible solution has an objective value far exceeding that from the heuristic algorithm. As such, the proposed heuristic algorithm outperforms the Gurobi method in terms of both solution quality and computation time, especially for large-scale problem instances.

For cases with the same network size and $R$ value, as the number of partitions $|J|$ increases, the computation time of our algorithm increases due to the larger number of variables and constraints. The objective value, however, reduces because with a larger number of partitions, the expected workload of each caller desk becomes smaller, and the probability of losing service due to insufficient adjacent districts is smaller. Similarly, a bigger value of $R$ results in a longer computation time (also because of the increase in problem size) and a smaller solution objective, probably due to the lower chance for any demand to lose service.

A closer look at some solutions is provided in Table 2, where the solution details for the cases with $n=8$ network, 7 partitions, and $R=3$ or 4 are presented. Column 3 lists the nodes in each district, Column 4 shows the total demand from all nodes in each district, and Column 5 gives the expected workload $X_{j}$ (re-)assigned to each caller desk $j$ in normal and disruption scenarios. Column 


\begin{tabular}{|c|c|c|c|c|c|c|c|}
\hline $\begin{array}{c}\text { Network } \\
\text { size }\end{array}$ & $\begin{array}{c}\text { Number of } \\
\text { partitions }\end{array}$ & $\begin{array}{c}\text { Number of } \\
\text { backup levels }\end{array}$ & $\begin{array}{l}\text { Heuristic } \\
\text { objective }\end{array}$ & $\begin{array}{c}\text { Heuristic } \\
\text { time }(\mathrm{s})\end{array}$ & $\begin{array}{c}\text { Gurobi } \\
\text { objective }\end{array}$ & $\begin{array}{l}\text { Gurobi } \\
\text { gap }\end{array}$ & $\begin{array}{l}\text { Gurobi } \\
\text { time }(\mathrm{s})\end{array}$ \\
\hline \multirow{4}{*}{$4 \times 4$} & 5 & 3 & 147.601 & 304 & 147.601 & $16.2 \%$ & 7200 \\
\hline & 5 & 4 & 146.699 & 374 & 145.707 & $21.7 \%$ & 7200 \\
\hline & 7 & 3 & 115.153 & 319 & 115.278 & $26.7 \%$ & 7200 \\
\hline & 7 & 4 & 102.642 & 505 & 119.778 & $31.9 \%$ & 7200 \\
\hline \multirow{4}{*}{$5 \times 5$} & 5 & 3 & 250.891 & 277 & 253.079 & $28.0 \%$ & 7200 \\
\hline & 5 & 4 & 242.446 & 630 & 260.021 & $34.6 \%$ & 7200 \\
\hline & 7 & 3 & 195.798 & 412 & 214.327 & $50.6 \%$ & 7200 \\
\hline & 7 & 4 & 174.914 & 856 & 223.066 & $66.1 \%$ & 7200 \\
\hline \multirow{4}{*}{$6 \times 6$} & 5 & 3 & 372.739 & 597 & 388.893 & $41.8 \%$ & 7200 \\
\hline & 5 & 4 & 362.828 & 1891 & 546.601 & $143.3 \%$ & 7200 \\
\hline & 7 & 3 & 297.416 & 1041 & 366.211 & $81.2 \%$ & 7200 \\
\hline & 7 & 4 & 269.098 & 2518 & 589.741 & $287.6 \%$ & 7200 \\
\hline \multirow{4}{*}{$7 \times 7$} & 5 & 3 & 528.513 & 3189 & 593.627 & $68.4 \%$ & 7200 \\
\hline & 5 & 4 & 521.621 & 4567 & 770.790 & $171.0 \%$ & 7200 \\
\hline & 7 & 3 & 422.193 & 3867 & 2311.332 & $740.3 \%$ & 7200 \\
\hline & 7 & 4 & 373.252 & 5845 & 2097.163 & $920.4 \%$ & 7200 \\
\hline \multirow{4}{*}{$8 \times 8$} & 5 & 3 & 727.619 & 3963 & - & - & - \\
\hline & 5 & 4 & 716.150 & 4327 & - & - & - \\
\hline & 7 & 3 & 575.618 & 4131 & - & - & - \\
\hline & 7 & 4 & 524.397 & 4697 & - & - & - \\
\hline
\end{tabular}

Table 1: Algorithm performance comparison for different test cases.

\begin{tabular}{cllccccccc}
\hline \multirow{2}{*}{ District } & Nodes & $\begin{array}{c}\text { Nodal } \\
\text { Demand }\end{array}$ & $\begin{array}{c}\text { Desk } \\
\text { Workload } X_{j}\end{array}$ & $C_{j}$ & 1 & 2 & 3 & 4 \\
\hline \multirow{6}{*}{$R=3$} & 1 & $44,45,51,52,53,59,60,61$ & 259.16 & 287.06 & 10.30 & 4 & 6 & 7 & - \\
& 2 & $25,26,33,34,41,42,43,49,50,57,58$ & 320.01 & 288.53 & 18.31 & 1 & 6 & 5 & - \\
& 3 & $8,15,16,22,23,24,31,32,40$ & 265.14 & 288.38 & 13.50 & 4 & 6 & 7 & - \\
& 4 & $3,4,5,6,7,13,14,21$ & 237.83 & 269.17 & 11.49 & 3 & 6 & 5 & - \\
& 5 & $1,2,9,10,11,12,17,18,19$ & 319.90 & 288.41 & 12.40 & 4 & 6 & 2 & - \\
& 6 & $20,27,28,29,30,35,36,37,38$ & 274.79 & 283.73 & 13.31 & 1 & 4 & 3 & - \\
& 7 & $39,46,47,48,54,55,56,62,63,64$ & 312.25 & 283.59 & 14.51 & 3 & 6 & 1 & - \\
\hline & 1 & $32,40,46,48,53,54,55,56,62,63,64$ & 312.45 & 289.14 & 20.32 & 5 & 2 & 4 & 7 \\
& 2 & $4,5,6,7,8,14,15,16,23,24$ & 301.36 & 274.36 & 17.41 & 5 & 1 & 6 & 3 \\
& 3 & $1,2,3,9,10,17,18,25,26,33,41$ & 303.55 & 275.89 & 21.31 & 6 & 4 & 2 & 7 \\
& 4 & $27,34,35,36,42,44,45$ & 256.02 & 284.03 & 10.30 & 6 & 5 & 3 & 1 \\
& 5 & $22,30,31,37,38,39,47$ & 256.04 & 288.20 & 9.39 & 6 & 1 & 4 & 2 \\
& 6 & $11,12,13,19,20,21,28,29$ & 238.08 & 287.95 & 10.30 & 4 & 3 & 5 & 2 \\
& 7 & $43,49,50,51,52,57,58,59,60,61$ & 321.57 & 289.47 & 16.22 & 4 & 1 & 3 & - \\
\hline
\end{tabular}

Table 2: Detailed results of the $8 \times 8$ network partitioned into 7 districts. 
6 presents compactness metric $C_{j}$ for each district. Columns 7-10 are the backup desk assignment decisions for each district at various backup levels. We observe that our proposed algorithm is able to achieve all partitioning objectives satisfactorily. Even though the original nodal demand from the districts are not quite even, the expected workloads (re-)assigned to all the desks are very well balanced. Figure 3 graphically illustrates the spatial partitions, where each connected subgraph represents one district. In general all the districts have reasonable compactness. Moreover, we observe that when $R=4$, districts in the partition results are slightly more elongated in shape than those when $R=3$, so as to have more adjacent districts (and hence, caller desks) as backup options. Specifically, when $R=3$, all districts but one have exactly 3 adjacent districts, while in the case when $R=4,6$ out of the 7 districts have at least 4 adjacent districts.

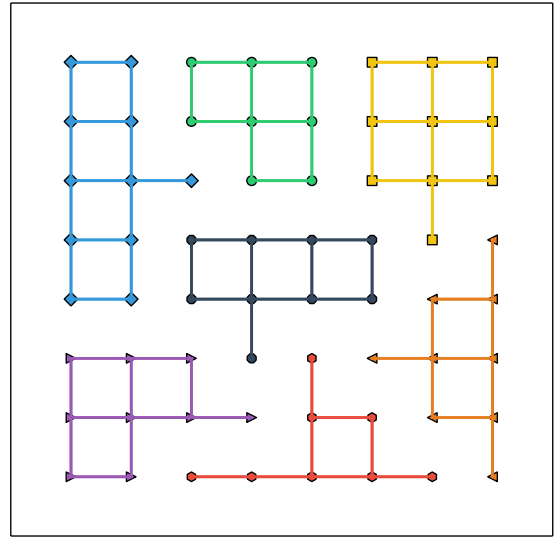

(a) $R=3$

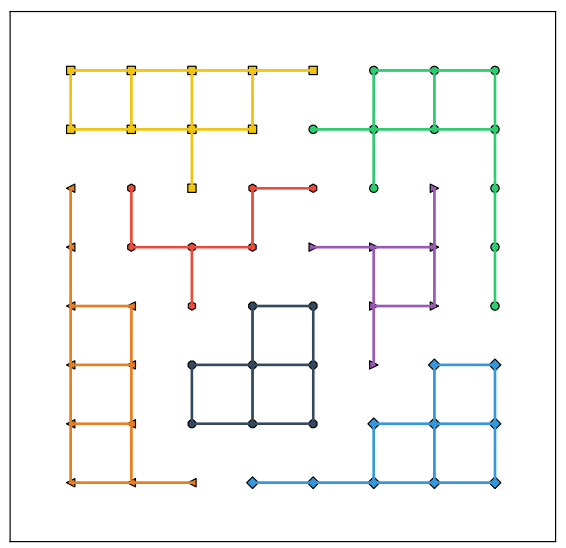

(b) $R=4$

Figure 3: The partition results of the $8 \times 8$ network under different values of $R$.

\subsection{Full-scale application}

Next, we apply our methodology to an empirical caller districting problem for a U.S. Class I railroad company. As shown in Figure 4, the railroad network consists of 157 nodes (the rail yards/supply points of the company), each with a specified demand of crew calls. The detailed data are confidential, but the maximum, minimum, and average of the nodal demand are 3219, 6 , and 76, respectively, representing a huge disparity in demand quantity across nodes. The distance between two adjacent nodes is measured along the shortest path in the actual rail track network. The company's call center contains 12 caller desks. Thus the company wants the network to be partitioned into $|J|=12$ corresponding districts and assigned to these desks. The maximum number of back levels is $R=3$, the probability for each desk to fail is $q=0.1$, and the penalty values are set as $W_{\text {balance }}=1.0, W_{\text {compact }}=2.0, W_{\text {missing }}=500.0$, respectively.

\begin{tabular}{ccccccccccccc}
\hline Index & 1 & 2 & 3 & 4 & 5 & 6 & 7 & 8 & 9 & 10 & 11 & 12 \\
\hline Demand & 9320 & 10240 & 9862 & 10239 & 10734 & 10970 & 8593 & 9747 & 9855 & 9635 & 10386 & 10261 \\
Workload & 10199 & 10160 & 9961 & 10182 & 9847 & 9995 & 9478 & 10012 & 9988 & 9639 & 10195 & 10175 \\
Level 1 & 11 & 12 & 1 & 9 & 4 & 8 & 10 & 7 & 3 & 7 & 2 & 1 \\
Level 2 & 5 & 10 & 9 & 3 & 3 & 9 & 8 & 6 & 8 & 8 & 5 & 10 \\
Level 3 & 12 & 3 & 6 & 5 & 11 & 10 & 6 & 9 & 6 & 6 & 12 & 2 \\
\hline
\end{tabular}

Table 3: The demand, workload, and backup assignments of each district. 


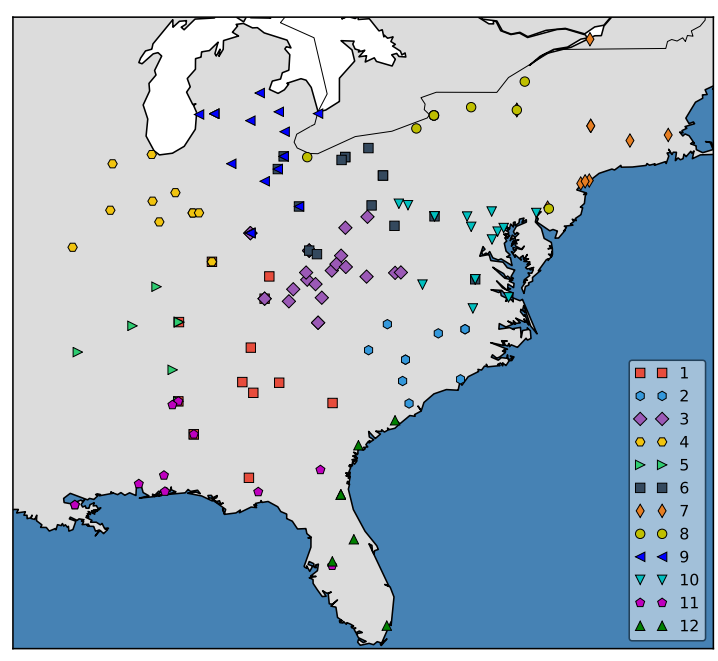

(a) Partition results

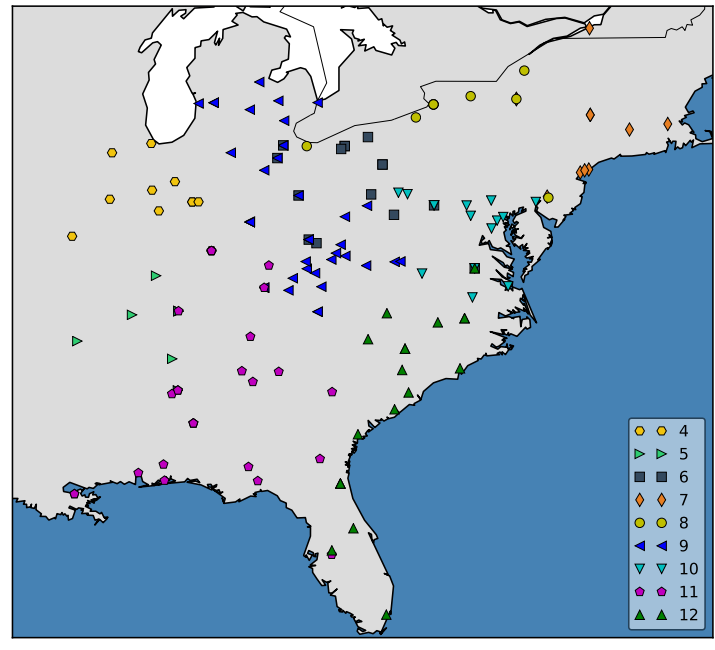

(b) Partition results when desks 1, 2, and 3 fail

Figure 4: Full-scale railroad caller districting problem.

The commercial solvers cannot solve a problem of this size. Results from the proposed heuristic algorithm is presented in Figure 4(a) and Table 3. In Figure 4(a), each color (or shape) of nodes corresponds to one district, which is contiguous and compact in shape in the company's network (although the detailed network is not shown). Table 3 shows the total nodal demand from each district and the expected workload (re-)assigned to each desk. The expected workloads of the desks are very well balanced, i.e., the biggest and smallest workloads are 10199 and 9478, respectively, with a mere $7.6 \%$ difference. Also, the $R=3$ choices of backup desks of each district are summarized in Table 3. For example, nodal demands in district 1 are assigned to desks 11, 5, and 12 at backup levels 1, 2, and 3, respectively. Figure 4(b) then shows the assignments of nodal demands to desks when desks 1, 2, and 3 fail. We observe that nodes in the original districts 1, 2, and 3 are reassigned to desks 11 (1st backup of district 1), 12 (1st backup of district 2), and 9 (2nd backup of district 3 since its 1st backup is disrupted already), respectively. The company considers such a result far superior than their current practice, and our model solution has since been implemented.

\begin{tabular}{cccccc}
\hline Number of partitions $|J|$ & Time (s) & Heuristic objective & $X_{\max }$ & $X_{0}$ & $\sum_{j \in J} C_{j}$ \\
\hline 8 & 2381 & 27335 & 15075 & 11.984 & 3134 \\
10 & 5117 & 23271 & 12065 & 11.984 & 2607 \\
12 & 7200 & 20883 & 10199 & 11.984 & 2346 \\
14 & 7200 & 19019 & 8703 & 11.984 & 2162 \\
16 & 7200 & 17709 & 7595 & 11.984 & 2061 \\
\hline Probability $q$ & Time (s) & Heuristic objective & $X_{\max }$ & $X_{0}$ & $\sum_{j \in J} C_{j}$ \\
\hline 0.025 & 6983 & 15019 & 10065 & 0.047 & 2465 \\
0.05 & 6454 & 15354 & 10137 & 0.749 & 2421 \\
0.1 & 7200 & 20883 & 10199 & 11.984 & 2346 \\
0.2 & 7045 & 111044 & 10048 & 191.747 & 2561 \\
\hline
\end{tabular}

Table 4: Sensitivity analysis on system parameters $|J|$ and $q$.

Finally, we conduct sensitivity analysis on two key system parameters: the number of partitions 

performance, as the number of partitions $|J|$ increases, the size of the problem becomes larger, and a longer computation time (i.e., up to 7200 seconds) is needed. As for each component of the objective, the maximum desk workload $X_{\max }$ and the compactness measure $\sum_{j \in J} C_{j}$ both decrease with $|J|$, while $X_{0}$ remains the same. This is intuitive. When $|J|$ is larger, the area size and average demand of each district are smaller, implying too that the districts are more compact. However, since the penalty for missing demand is high, the demand of every district will always be assigned to the dummy desk (pay the penalty) at the highest possible level $R+1=4$ with the smallest possible probability $q^{4}=0.0001$. This contributes to the same value of $X_{0}$ under different values of $|J|$. On the other hand, as the probability value $q$ increases, the computational time does not change much due to the invariant problem size. The values of $X_{\max }$ and $\sum_{j \in J} C_{j}$ also almost remain the same under the same number of partitions $|J|$. However, the value of $X_{0}$ increases significantly because a larger value of $q$ leads to a higher probability for the demand to lose service (and hence, bear the penalty).

\section{Conclusion}

In this paper, we studied a reliable caller districting problem, motivated by the current railroad practice, which aims to partition an undirected network into a fixed number of districts and assign their demands to different caller desks. Several operational criteria including district contiguity and compactness, caller desk service reliability, and workload balance are explicitly addressed in a mixed-integer programming model. Customized solution approaches including a constructive heuristic and a neighborhood search algorithm are designed to obtain near-optimum solutions. We conduct several numerical examples (including an empirical case study) with different network sizes and parameter settings to demonstrate the applicability and performance of our methodology. Results of the examples show that the proposed heuristic algorithm outperforms commercial solver Gurobi in terms of providing good quality solutions in a shorter amount of computation time. In addition, managerial insights are drawn from the various numerical examples.

Future research can be conducted in several directions. First, real world problems may involve additional types of exogenous factors on the caller desks; it would be desirable to consider more general desk failure patterns, e.g., the failure probabilities of caller desks are site-dependent (Cui et al., 2010) or the failures are spatially correlated (Xie et al., 2015a). Second, although the proposed heuristic algorithm is capable of providing solutions with good quality, no accuracy information about the solutions is provided. It will be very interesting to explore other customized algorithms (e.g., column generation, Lagrangian relaxation) for large-scale applications. Furthermore, the reliable caller districting problem could be potentially extended to many other contexts. For example, one possible extension is a variant of the multi-depot location-routing problem, in which each depot sends vehicles to serve the demands in one district, and the vehicles are only allowed to move within the assigned district (Xie et al., 2015b). We can also study the districting problem in a continuous setting; i.e., by replacing the discrete network with a continuous plane, and find general rules for partitioning a continuous plane with the consideration of reliability issues (Li and Ouyang, 2010; Wang et al., 2015).

\section{Acknowledgments}

This research was financially supported in part by CSX Transportation and by the U.S. National Science Foundation through Grant CMMI \#1234085. 


\section{References}

Berman, O., Krass, D., B.C., M., 2007. Facility reliability issues in network p-median problems: Strategic centralization and co-location effects. Operations Research 55 (2), 332-350.

Blais, M., Lapierre, S. D., Laporte, G., 2003. Solving a home-care districting problem in an urban setting. The Journal of the Operational Research Society 54 (11), 1141-1147.

Bozkaya, B., Erkut, E., Laporte, G., 2003. A tabu search heuristic and adaptive memory procedure for political districting. European Journal of Operational Research 144 (1), 12-26.

Camacho-Collados, M., Liberatore, F., Angulo, J., 2015. A multi-criteria police districting problem for the efficient and effective design of patrol sector. European Journal of Operational Research $246(2), 674-684$.

Chen, Q., Li, X., Ouyang, Y., 2011. Joint inventory-location problem under the risk of probabilistic facility disruptions. Transportation Research Part B: Methodological 45 (7), 991-1003.

Cui, T., Ouyang, Y., jun Max Shen, Z., 2010. Reliable facility location design under the risk of disruptions. Operations Research 58 (4), 998-1011.

Ferland, J. A., Guénette, G., 1990. Decision support system for the school districting problem. Operations Research 38 (1), 15-21.

Garfinkel, R. S., Nemhauser, G. L., 1970. Optimal political districting by implicit enumeration techniques. Management Science 16 (8), B495-B508.

Hess, S. W., Samuels, S. A., 1971. Experiences with a sales districting model: Criteria and implementation. Management Science 18 (4-part-ii), 41-54.

Hess, S. W., Weaver, J. B., Siegfeldt, H. J., Whelan, J. N., Zitlau, P. A., 1965. Nonpartisan political redistricting by computer. Operations Research 13 (6), 998-1006.

Li, X., Ouyang, Y., 2010. A continuum-approximation approach to reliable facility location design under correlated probabilistic failures. Transportation Research Part B: Methodological 44 (4), $535-548$.

Mehrotra, A., Johnson, E. L., Nemhauser, G. L., 1998. An optimization based heuristic for political districting. Management Science 44 (8), 1100-1114.

Muyldermans, L., Cattrysse, D., Oudheusden, D. V., Lotan, T., 2002. Districting for salt spreading operations. European Journal of Operational Research 139 (3), 521-532.

Sherali, H., Alameddine, A., 1992. A new reformulation-linearization technique for bilinear programming problems. Journal of Global Optimization 2 (4), 379-410.

Snyder, L. V., Daskin, M. S., 2005. Reliability models for facility location: The expected failure cost case. Transportation Science 39 (3), 400-416.

Wang, X., Lim, M. K., Ouyang, Y., 2015. A continuum approximation approach to the dynamic facility location problem. Transportation Science, In press.

Xie, S., Li, X., Ouyang, Y., 2015a. Decomposition of general facility disruption correlations via augmentation of virtual supporting stations. Transportation Research Part B: Methodological $80,64-81$. 
Xie, W., Ouyang, Y., Wong, S. C., 2015b. Reliable location-routing design under probabilistic facility disruptions. Transportation Science 50 (3), 1128-1138.

8 